\title{
Identification of Requirements using Goal Oriented Requirements Elicitation Process
}

\author{
Mohd. Arif \\ M. Tech. Scholar, Department of Computer \\ Science and Engineering, Al-Falah School of \\ Engineering and Technology, Dhauj, Faridabad, \\ Haryana, India
}

\author{
Saoud Sarwar \\ Department of Computer Science, Al-Falah \\ University, Faridabad, Haryana, India
}

\begin{abstract}
Goal Oriented Requirements Elicitation Process (GOREP) is used to identify the requirements of software using AND/OR graph. Requirements elicitation plays an important role for the identification of software's functional and non-functional requirements. The objective of this paper is twofold; firstly, we classify different requirements elicitation techniques on the basis of different criterion like traditional, cognitive, collaborative, and contextual techniques; and secondly, we integrate mind map with those nodes of AND/OR that we want to further explore at the time of requirements elicitation process. Finally, the utilization of integrating the mind map with AND/OR graph is demonstrated with the help of an example.
\end{abstract}

\section{Keywords}

Requirements Elicitation Process, GOREP, Functional and Non-Functional Requirements.

\section{INTRODUCTION}

Requirements elicitation is an important sub-process of requirement engineering [15]. It is the process of searching, uncovering, achieving, and detailing requirements for different type of systems like computer based systems, web based systems etc. [10]. Requirements elicitation is all about attainments and understanding the needs of users and project promoters with the ultimate aim of communicating these needs to the system developers [16]. It also commits a set of activities that must allow for communication, prioritization, consultation, and collaboration with the entire relevant stakeholders [10]. In requirements elicitation process, requirements are analyzed as the main resources, and also on the basis of accurate analysis of the organization, the application area where the system will be disposed [13].

The objective of this paper is twofold: Firstly, we extend the classification scheme of [15]. In our classification, we classify the requirements elicitation techniques on the basis of traditional, cognitive, collaborative, and contextual techniques (See Fig.1). Traditional Techniques are used to identify the limitation of the current system by using different methods like interview, questionnaire, analysis of existing documents etc. Cognitive technique includes task analysis, protocol analysis, and knowledge acquisition technique etc. Collaborative techniques are used for different activities like selection and prioritization of software requirements [18], detection of discordances among stakeholders etc. In Contextual technique, requirements are identified at the work place of the customer. Ethnographic, discourse analysis, socio-technical are some of the important methods of contextual technique. Secondly, we integrate mind-map method with those nodes of AND/OR graph that we want to further at the time of requirements elicitation process. This paper is organized as follows: In Section II, we explain our classification scheme. Section III, presents an extended version of GOREP by integrating the mind-map with AND/OR graph. An example is provided in section IV. Finally, conclusion and future work is given in section V.

\section{CLASSIFICATION SCHEME}

In this section, we present our classification scheme of requirements elicitation techniques (See Fig. 1). In this classification, we have four sub-classifications like traditional, cognitive, collaborative, and contextual techniques. In the following sub-section, we explain our classification scheme.

(A) Traditional techniques are used to identify the limitation of the current system. These limitations are considered as the set of requirements for the proposed system. We divide traditional techniques into following parts i.e. introspection, reading existing documents, analyzing hard data, interviews, surveys/questionnaires, and meetings. Brief descriptions of these techniques are given below:

(i) Introspection is the first and most obvious method for trying to understand what properties a system should have in order to succeed [10]. It is mainly used only as a starting point for other requirements elicitation efforts [7].

(ii) Reading Existing Document Technique is used to elicit the requirements of a current system by studying present documentation and identifying significant information [13].

(iii) Analyzing Hard Data is used to explore the forms, invoices, reports to gather the facts, figures, survey results, financial information, marketing data and reports for decision making [14].

(iv) Interviews are most common professional analysis techniques to identify requirements, verify the facts, engage end users, clarify ambiguity, trigger enthusiasm and solicit opinions and ideas. In literature, we have identified that interview is the most promising approach of requirements elicitation [5]. 


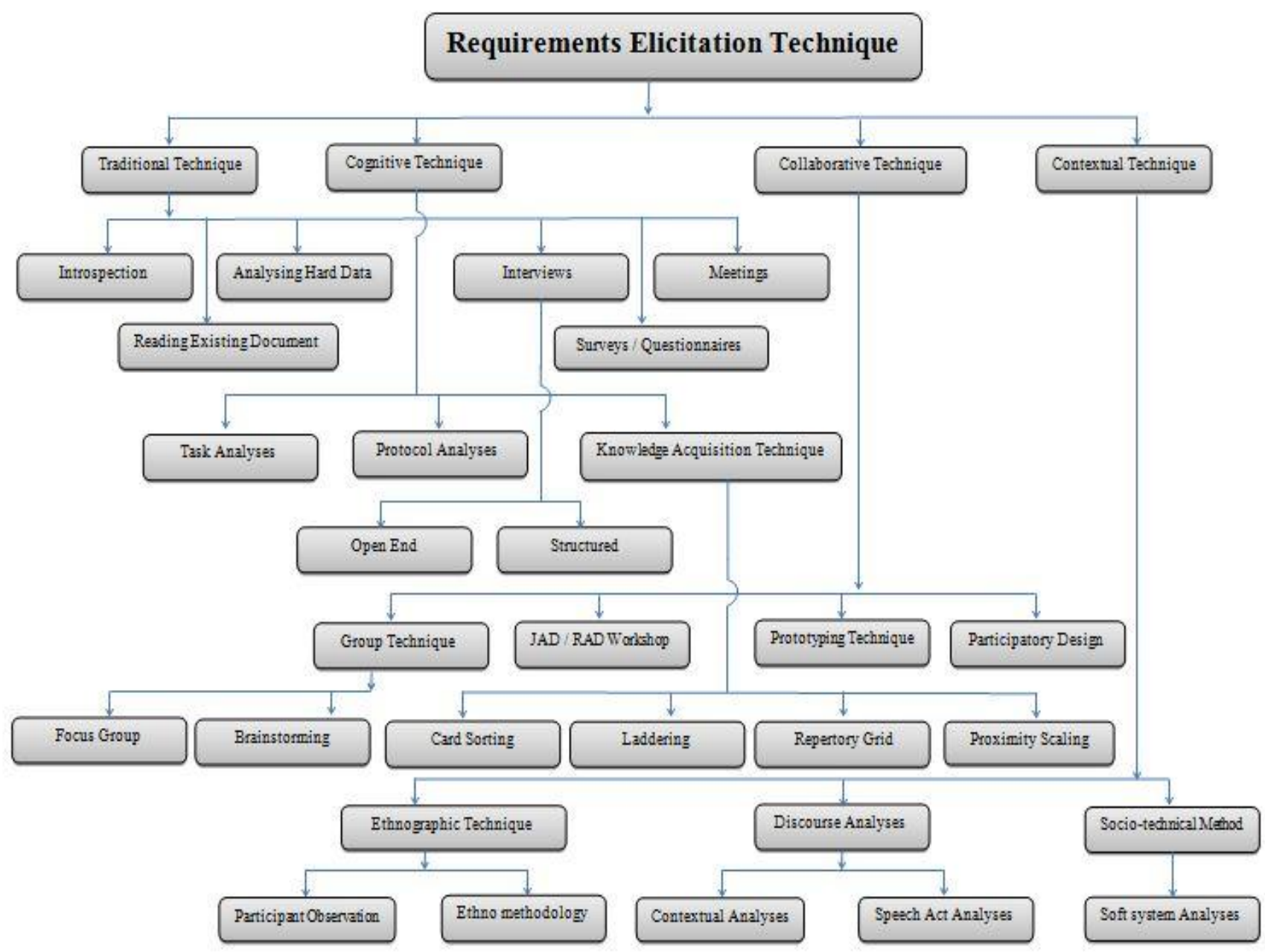

Fig. 1 Classification of Requirements Elicitation Techniques

(v) Surveys / Questionnaires are used for eliciting information from many people, anonymously, in a relatively short time. A survey is often referred to as a questionnaire to collect information about customers, products, work practices and attitudes [8].

(vi) Meeting can be categories into formal or informal meetings. These techniques are particularly useful in case of conflicts among different stakeholders.

(B) Cognitive Techniques are based on multidisciplinary methodologies as the information on knowledge elicitation methods is widely scattered across the fields of knowledge engineering, anthropology, psychology, education, business management, counseling, cognitive science, linguistics, and philosophy [6]. There are different techniques like task analysis, protocol analysis, and knowledge acquisition technique.

(i) Task Analysis technique used to multiple varieties of interviews and observation strategies to capture a description of the knowledge that experts use to perform complex tasks. [6].

(ii) Protocol Analysis is used to analyze protocols to reveal requirements. This technique is based on the protocols created from vocalizing behavior either 'think-aloud' or retrospective. The major advantages are direct verbalization of cognitive activities, embedded in the work context [6].

(iii) Knowledge Acquisition Technique is employed to gain knowledge using several methods like card sorting, laddering, repertory grid, and proximity scaling. Brief descriptions of these techniques are given below:

(a) Card Sorting is a recognized method for requirements elicitation. It has been established that card numbering has many affirmative phases that makes it efficient tool for requirement elicitation [11].

(b) Laddering is a structured questioning technique derived from the repertory grid technique, enabling a hierarchy of concepts to be established. Laddering is best fit for integration with a protocol editor in which the output of an editor is used as a conceptual input [2].

(c) Repertory Grid is a simple knowledge elicitation technique normally used by clinical psychologists after identifying a small set of elements. The method also helps to elicit an initial representation, which can afterwards be refined by applying other established refinement mechanisms [6].

(d) In Proximity Scaling a set of dimensions are derived for classifying domain objects. It includes two steps, i.e. (i) Pair wise proximity assessment among domain elements, which captures tacit knowledge of expert. (ii) Afterwards, automated statistical analysis is done to build multi-dimensional space to classify the objects. Major advantages are that it helps to elicit mental models where complex multivariate data is concerned and it is good for eliciting tacit knowledge also. However, it requires an agreed-upon set of objects and only models classification knowledge, not performance knowledge [6]. 
(C) Collaborative Techniques are used for the selection and prioritization of requirements in which several stakeholders participate during requirements elicitation process. There are different collaborative techniques like group technique, JAD/RAD workshops, prototyping, and participatory design [8].

(i) Group Technique involves participation of different stakeholders. It includes the following: Focus group and Brainstorming technique to achieve the goal [15].

(a) Focus Group is a means to elicit ideas and attitudes about a specific product, service or opportunity in an interactive group environment. The participants share their impressions, preferences and needs, guided by a moderator [7].

(b) Brain Storming is an excellent way of eliciting many creative ideas for an area of interest. Structured brainstorming produces numerous creative ideas about any given "central question" or topic [8].

(ii) JAD / RAD Workshop Joint Application Development (JAD) and Rapid Application Development (RAD) accentuate user involvement through group meetings with balanced facilitator [12].

(iii) Prototyping is used as an elicitation technique, aims to uncover and visualize interface requirements before the application is designed or developed.

(iv) Participatory Design represents an approach in which the users of a system play a critical role in the design process [3]. Through participatory design techniques, the design team is also able to incorporate the data provided by the users through continuous user involvement. The users are asked to participate in those aspects of the project where their input is viewed as valuable, but are left out of most technology-related discussions [4].

(D) Contextual Techniques are used to identify the requirements at the workplace of the customers. There are different method of contextual technique like ethnography, discourse analysis, and socio-technical method.

(i) Ethnographic Technique is a social science elicitation technique that supports context-aware technique requirements. The fundamental basis of ethnography is that people's actions and thoughts are dependent on a vast range of factors [4]. This technique is further sub-divided into participant observation and ethno-methodology.

(a) Participant Observation means to elicit requirements by conducting an assessment of the subject matter expert's work environment. This technique is appropriate when documenting details about the current processes or if the project intends to enhance or change a current process.

(b) Ethnographic Driven by the 'failure' of systems that manifestly did not meet the needs of their users, fieldwork is an approach to the study of work where an observer engages directly with work in its own environment $[17,4]$.

(ii) Discourse Analysis is an extended version of ethnography techniques. It consists of two sub techniques called Conversation Analysis and Speech Act Analysis. Discourse analysis offers various tools to examine systematically the fundamental substance of requirements elicitation i.e. interactional talk. This analysis employs sociolinguistic methods for specifying the linguistic features of different types of discourse units and the way they are tied together to create meaning, with critically examining social context [10]. (iii) Socio-technical Method produces informal models of a socio-technical system. The primary consideration in these techniques is the system, people and the organization. Rather, they are ways of understanding a problem and its organizational context. Software Systems Methodology (SSM) is probably the best known of these methods. The essence of SSM is its recognition that systems are embedded in a wider human and organizational context [4].

\section{INTEGRATING MIND MAP WITH GOREP}

In order to strengthen the goal oriented requirement elicitation process (GOREP) [17], in [1] we proposed a method for integrating the Mind Map (MM) [9] with AND/OR Graph. MM is a thinking tool which reflects how information's are stored in the brain as well as how information's are retrieved from the brain of stakeholders. It is an effective method of expressing individual ideas and its associations in an organized way [1]. In GOREP, AND/ OR graph is constructed by decomposing the high level objective of stakeholders into sub goals or requirements. This graph is used to identify the functional and non functional requirements. In AND composition, if all of the goals or requirements are achieved, their parent goals can be achieved or satisfied. On the other hand side, in OR decomposition, the achievement of at least one sub goals or requirements leads to the achievement of its parent goal [18]. The proposed method is presented as follows [1]: (a) Identify stakeholders [18], (b) Identify high level objective of the primary stakeholder using any requirements elicitation technique (See Fig. 1). (c) Construct AND/OR graph, (d) Attach MM with each node of AND/OR graph, and (e) then Classify functional requirements (FR) and non functional requirements (NFR).

\section{AN EXAMPLE}

In-order to show, how the propose method works, in this section we consider the problem of Institute Examination System (IES), adopted from the work of [16]. This system is used to provide the facility to submit online examination form and generate the results of the students; and display the news related to examinations. After submitting the examination form, the system will generate the examination fee receipt and students will be able to take the printout of that receipt. The system will also generate the patterns of the sitting arrangement.

Step 1 and Step 2: Let us assume that four stakeholders are participating in requirements elicitation process. The responsibilities of these four stakeholders are given as below: $\mathrm{S}_{1} \rightarrow$ Responsible for elicitation of FR of Student's module $\left(\mathrm{fr}_{1}\right)$

$\mathrm{S}_{2} \rightarrow$ Responsible for elicitation of FR of Examination Management module $\left(\mathrm{fr}_{2}\right)$

$\mathrm{S}_{3} \rightarrow$ Responsible for elicitation of FR of Examiner's module $\left(\mathrm{fr}_{3}\right)$

$\mathrm{S}_{4} \rightarrow$ Responsible for elicitation of NFR of IES $\left(\mathrm{fr}_{4}\right)$

Step 3: Let us assume that during group requirements elicitation process, requirements analyst collect the requirements from different stakeholders and construct the AND/OR graph by decomposing the objective of stakeholders into three FR, i.e., student module, examination management module, and examiner's module; and there is one nonfunctional requirement, i.e., Trustworthiness (see Fig. 2). Trustworthiness means that the system should have one or more of the following characteristics: safety, security, performance etc. In Fig. 2, Student module, examination management module, and examiner's modules are represented 
by $\mathrm{FR}_{1}, \mathrm{FR}_{2}$, and $\mathrm{FR}_{3}$ respectively; and trustworthiness is represented by NFR. There is an AND decomposition among $\mathrm{FR}_{1}, \mathrm{FR}_{2}, \mathrm{FR}_{3}$ and NFR as shown in Fig. 2. $\mathrm{FR}_{1}, \mathrm{FR}_{2}$, and $\mathrm{FR}_{3}$ are further decomposed and refined into twenty nine FRs, i.e., $\mathrm{fr}_{1}$ (Students), $\mathrm{fr}_{1.1}$ (Fees of the student), $\mathrm{fr}_{1.1 .1}$ (Submit online fees), $\mathrm{fr}_{1.1 .2}$ (Submit offline fees), $\mathrm{fr}_{1.1 .2 .1}$ (Receipt number of the fees submitted), $\mathrm{fr}_{1.1 .2 .2}$ (Date of the fees submitted), $\mathrm{fr}_{1.2}$ (Sessional marks of the student), $\mathrm{fr}_{1.2 .1}$ (Theory), $\mathrm{fr}_{1.2 .2}$ (Practical), fr $\mathrm{fr}_{1.3}$ (Student detail), fr. $\mathrm{fr}_{1.3 .1}$ (Student name), fr 1.3.2 $_{1}$ (Student roll number), fr $r_{1.3 .3}$ (Student enroll number), $\mathrm{fr}_{2}$ (Examination), $\mathrm{fr}_{2.1}$ (Theory), $\mathrm{fr}_{2.1 .1}$ (Paper Setter), $\mathrm{fr}_{2.1 .1 .1}$ (Internal Examiner), $\mathrm{fr}_{2.1 .1 .2}$ (External Examiner), $\mathrm{fr}_{2.1 .2}$ (Paper evaluator), $\mathrm{fr}_{\text {2.1.2.1 }}$ (Internal Examiner), $\mathrm{fr}_{\text {2.1.2.2 }}$ (External Examiner), fr ${ }_{2.2}$ (Practical), $\mathrm{fr}_{2.2 .1}$ (Internal Examiner), $\mathrm{fr}_{2.2 .2}$ (External Examiner), $\mathrm{fr}_{3}$ (Examination system management), $\mathrm{fr}_{3.1}$ (Printing Section), $\mathrm{fr}_{3.2}$ (Faculties and Centers), $\mathrm{fr}_{3.3}$ (Tabulators), $\mathrm{fr}_{3.4}$ (Exam Controller). In Fig. 3, we draw mind map of Sessional marks of students, because we want to further explore the "Sessional marks" nodes of students of AND/OR graph.

\section{CONCLUSION AND FUTURE WORK}

Requirements elicitation is an important sub-process of requirements engineering. Error occurring during requirements elicitation will affect the remaining subprocesses like requirements modeling, requirements analysis, requirements verification and validation, and requirements management. In this paper, first classified various requirements elicitation techniques, so that at the time of requirements elicitation, requirements analyst can easily identify an effective technique according to their needs. In order to strengthen the requirements elicitation process, in this paper we integrate mind map with those nodes of AND/OR graph that we want to further explore. Future research work includes the following agenda.

1. Classification of goal oriented requirements elicitation processes.

2. To apply the proposed method for constructing the AND/OR graph for different modules of institute examination system like examiner module and examination management module.

\section{REFERENCES}

[1] Shahnawaz A and Sadiq M, "Goal Oriented Mind Map Generation Method for Requirements Elicitation Process", IEEE International Conference on "Futuristic Trends in Computational analysis and Knowledge management," (A-BLAZE), organized by Amity University, Greater Noida, India 2015 (accepted for publication).

[2] Corbridge C, Rugg, G., Major, N.P., Shadbolt, N.R. and Burton, A.M. "Laddering technique and tool use in knowledge acquisition. Knowledge Acquisition," 6.pp.315- 341, 1994.

[3] Morkos B, "Elicitation and Development of Requirements Through Integrated Methods," DETC2009-87720.

[4] F. Kensing and J. Blomberg, "Participatory Design: Issues and Concerns," in Computer Supported Cooperative Work: Kluwer Academic Publishers, pp. 167-185, 1998.

[5] Dieste $\mathrm{O}$ and Juristo $\mathrm{N}$, "Systematic review and Aggregation of Empirical Studies on Elicitation Techniques," IEEE Transactions on Software
Engineering, Vol. 37, No. 2 pp. 283-304, 2011.

[6] Sharma S and Pandey S.K, "Revisiting Requirements Elicitation Techniques," International Journal of Computer Applications (0975-8887), Vol. 75, No. 12, August 2013.

[7] Sadiq M, G Shabina ,Shahid M, "An Approach for Eliciting Software Requirements and its Prioritization using Analytic Hierarchy Process", IEEE International Conference on Advances in Recent Technologies in Communication and Computing, pp.790-795 Kerala, India, October 2009.

[8] Sadiq M, A Jawed, Asim M, Qureshi A, Suman R, “ More on Elicitation of Software requirements and prioritization using AHP", IEEE International Conference on Data Storage and Data Engineering pp 232-236, Bangalore, India, February 2010.

[9] Jaafar Juliana and Atan Mislina, "Collaborative Mind Map tool to Facilitate Requirements Elicitation," "Proceeding of the $3^{\text {rd }}$ International Conference on Computing and Informatics (ICOCI)," 8-9 June, Bandung, Indonesia.

[10] Zowghi Didar and Coulin Chad, "Requirements elicitation: "A survey of Techniques, Approaches, and Tool,", "Engineering and Managing Software Requirements," Springer, pp. 19-46, 2005.

[11] Kotonya, G. and Sommerville, I.,"Requirements Engineering, Process and Techniques", Willy New York 1998.

[12] Nurmuliani, Nur. Zowghi, Didar and Susan P. Williams 2009. Using Card Sorting Technique to Classify Requirements Change.

[13] Requirements Elicitation: A Survey of Techniques, Approaches, and Tools.

[14] Sadiq M and Jain SK "A Fuzzy Based Approach for the Selection in Goal Oriented Requirements Elicitation Process," International Journal of System Assurance Engineering and Management, Springer, 2014

[15] Sadiq M and Jain SK "An Insight into Requirements Engineering Processes," International Conference of Advance in Communication, Networking and Computing (CNC), LNCSIT, Springer, PP. 313-318, 2012 India.

[16] Sadiq M and Jain SK “Applying Fuzzy Preference relation for Requirements Prioritization in Goal Oriented Requirements Elicitation Process," International Journal of System Assurance Engineering and Management, Springer Vol. 5 Issue 4 pp. 711-723, 2014.

[17] Sadiq M and Jain SK, "A Fuzzy Based Approach for Requirements Prioritization in Goal Oriented Requirements Elicitation Process," International Conference of Software Engineering and Knowledge Engineering (SEKE), pp. 54-58, 2013 USA.

[18] Sadiq M and Jain SK, "Stakeholders Identification Methods in Goal Oriented Requirements Elicitation Process," Fifth IEEE International Workshop on Requirements Prioritization and Communication at IEEE International Requirements Engineering Conference (RE), pp. 25-33, 2014 Karlskrona, Sweden. 


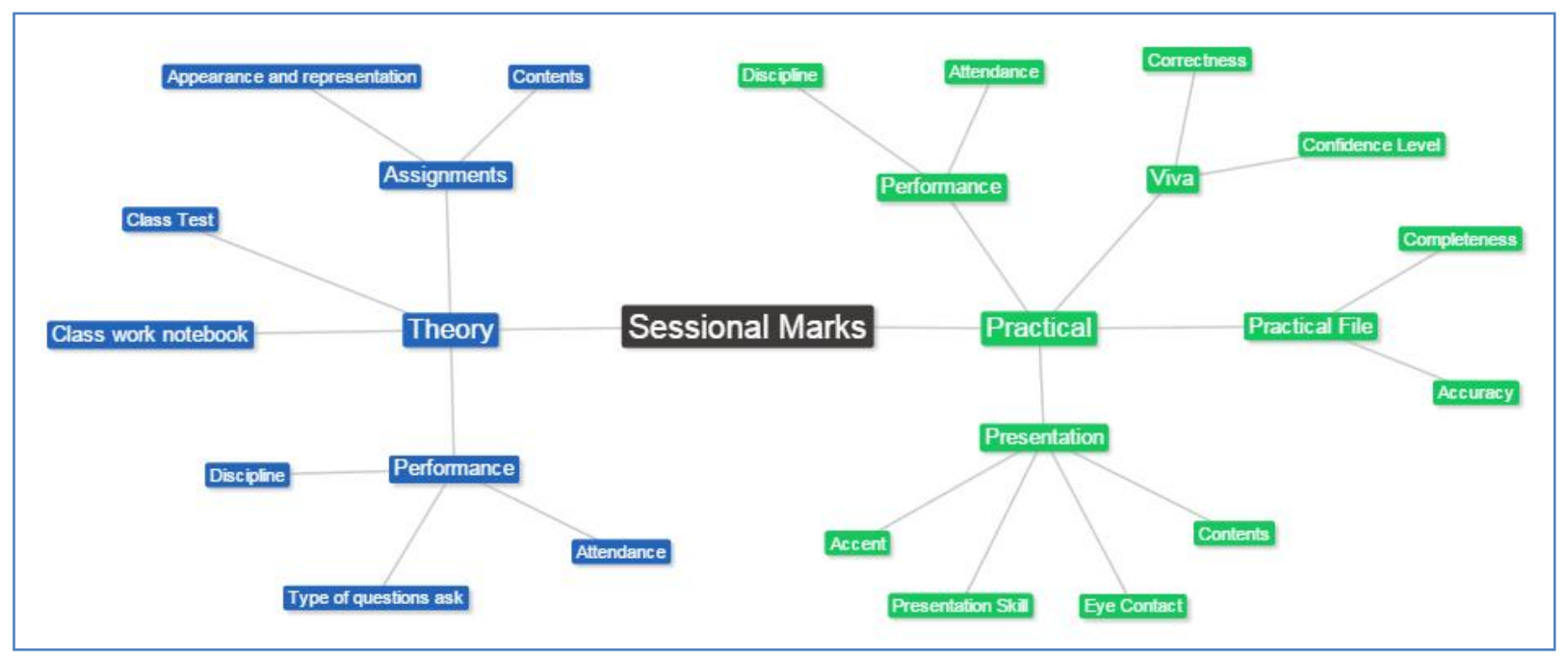

Fig. 3 Mind Map for Sessional Marks

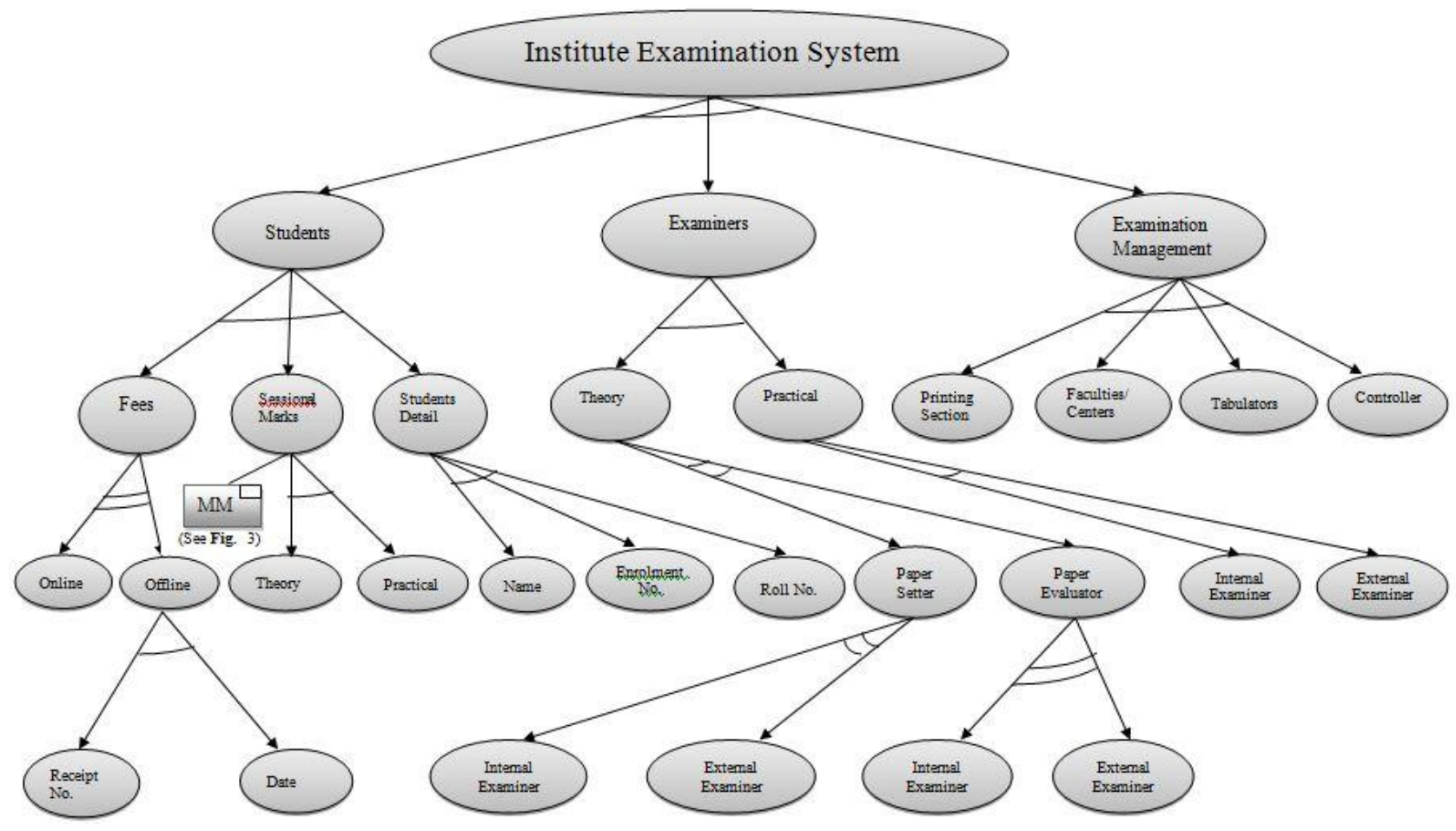

Fig. 2 AND/OR graph for Institute Examination System 